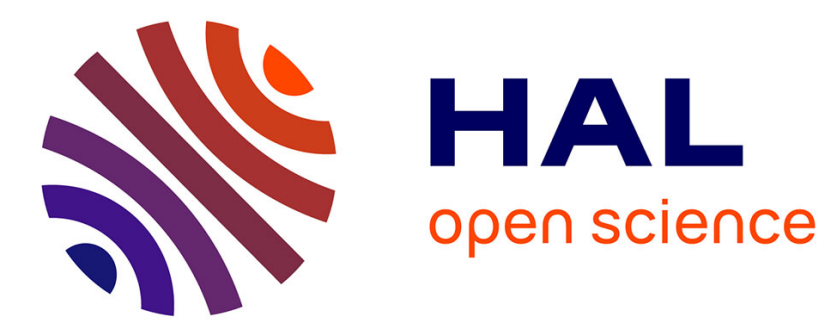

\title{
Congruence lattices of free lattices in non-distributive varieties
}

Miroslav Ploscica, Jiri Tuma, Friedrich Wehrung

\section{To cite this version:}

Miroslav Ploscica, Jiri Tuma, Friedrich Wehrung. Congruence lattices of free lattices in nondistributive varieties. Colloquium Mathematicum, 1998, 76, no. 2, pp.269-278. hal-00004064

\section{HAL Id: hal-00004064 https://hal.science/hal-00004064}

Submitted on 25 Jan 2005

HAL is a multi-disciplinary open access archive for the deposit and dissemination of scientific research documents, whether they are published or not. The documents may come from teaching and research institutions in France or abroad, or from public or private research centers.
L'archive ouverte pluridisciplinaire HAL, est destinée au dépôt et à la diffusion de documents scientifiques de niveau recherche, publiés ou non, émanant des établissements d'enseignement et de recherche français ou étrangers, des laboratoires publics ou privés. 


\title{
CONGRUENCE LATTICES OF FREE LATTICES IN NON-DISTRIBUTIVE VARIETIES
}

\author{
MIROSLAV PLOŠČICA, JIŘÍ TŮMA, AND FRIEDRICH WEHRUNG
}

\begin{abstract}
We prove that for any free lattice $F$ with at least $\aleph_{2}$ generators in any non-distributive variety of lattices, there exists no sectionally complemented lattice $L$ with congruence lattice isomorphic to the one of $F$. This solves a question formulated by Grätzer and Schmidt in 1962. This yields in turn further examples of simply constructed distributive semilattices that are not isomorphic to the semilattice of finitely generated two-sided ideals in any von Neumann regular ring.
\end{abstract}

\section{INTRODUCTION}

One of the oldest and most famous open problems in lattice theory, the Congruence Lattice Problem, is to decide whether for every distributive (join-) semilattice $S$ with zero, there exists a lattice $L$ such that the semilattice $\mathbf{C}(L)$ of compact congruences of $L$ (the congruence semilattice of $L$ ) is isomorphic to $S$. Although the answer is not known yet, many partial results have been obtained (see [7] for a survey). Among these are positive solutions of the Congruence Lattice Problem in the case where $S$ has size at most $\aleph_{1}$, or is a distributive lattice. In addition, it turns out that in several cases, the solution lattice $L$ to the problem is sectionally complemented (e.g., for the finite case, see [3]; the case where $S$ is countable results from unpublished work of G. M. Bergman [1] and results in [9]). As there seems to be a growing evidence that in all known cases, there exists a sectionally complemented solution lattice $L$, one may be tempted to formulate the even stronger conjecture that every distributive semilattice with zero is isomorphic to the congruence semilattice of a sectionally complemented lattice. This conjecture had in fact already been formulated in [2, Problem II.8].

In [9], using a construction presented in [8], the third author proves that it cannot be so, by giving a distributive semilattice of size $\aleph_{2}$ that is not isomorphic to the congruence semilattice of any lattice that is, in the terminology of [9], congruence splitting. In particular, every lattice which is either sectionally complemented, or relatively complemented, or a direct limit of atomistic lattices, is congruence splitting. Therefore, if one could prove that for every lattice $L$ there exists a sectionally complemented (or, more generally, congruence splitting) lattice $K$ such

Date: January 25, 2005.

1991 Mathematics Subject Classification. Primary 06B10, 06B15, 06B20, 06B25; Secondary 16E50, 08A05, 04A20.

Key words and phrases. Congruence lattice, congruence splitting lattice, Uniform Refinement Property, Kuratowski's Theorem, diamond, pentagon.

The research of the first author was supported by Slovak GAV grant 1230/96.

The second author was partially supported by GAČR grant no. 201/95/0632 and by GA AV ČR grant no. A 1019508. 
that $\mathbf{C}(L) \cong \mathbf{C}(K)$, then one would obtain a negative solution to the Congruence Lattice Problem.

This turns out to be also an open problem, more specifically the second part of [3, Problem 1, p. 181]. In this paper we give a strong negative solution to this problem, by proving (Corollary 4.2 ) that in any non-distributive variety of lattices, if $F$ is any (bounded or not) free lattice with at least $\aleph_{2}$ generators, then there exists no congruence splitting lattice $L$ such that $\mathbf{C}(F) \cong \mathbf{C}(L)$; in particular, $F$ has no congruence-preserving embedding into any sectionally complemented lattice. By earlier results in [9], this implies that $\mathbf{C}(F)$ is never isomorphic to the semilattice of finitely generated two-sided ideals in a von Neumann regular ring. The restrictions on the lattice variety are optimal, because of the classical result that says that every distributive lattice embeds congruence-preservingly into a generalized Boolean algebra.

The strategy of the proof is the following: by the results of [9], the congruence semilattice of any congruence splitting lattice satisfies a certain infinite axiom, the Uniform Refinement Property (URP). In this paper, we introduce a slight weakening of URP, the weak Uniform Refinement Property (WURP), that is not satisfied by the congruence semilattice of any free lattice with at least $\aleph_{2}$ generators in any non distributive lattice variety $\mathcal{V}$. The two cases in which the proof splits, namely whether the diamond $M_{3}$ or the pentagon $N_{5}$ belongs to $\mathcal{V}$, are treated in a similar fashion: they are decorated with three 2-element chains that somewhat concentrate into a finite pattern the combinatorial core of the original infinite WURP. As in [8], the reduction of the infinite case to the finite case is done via Kuratowski's free set property ([5]; see also [8, Proposition 2.5] for a short proof).

\section{NOTATION AND TERMINOLOGY}

We consider semilattices of compact congruences of lattices. The semilattices are join semilattices with 0 . The mapping assigning to every lattice $L$ its congruence semilattice $\mathbf{C}(L)$ can be extended to a functor from the category of lattices and lattice homomorphisms to the category of semilattices with homomorphisms of semilattices; in addition, this functor preserves direct limits. The least and largest congruence on $L$ will be respectively denoted by $\mathbf{0}$ and $\mathbf{1}$.

For all elements $a$ and $b$ of a lattice $L$, we will denote by $\Theta(a, b)$ the least congruence containing the pair $(a, b)$ and we will then put $\Theta^{+}(a, b)=\Theta(a \wedge b, a)$; thus $\Theta^{+}(a, b)$ is the least congruence $\theta$ on $L$ such that $\theta(a) \leq \theta(b)$.

We say that a homomorphism of semilattices $\mu: S \rightarrow T$ is weak-distributive $[9$, Section 1] when for all $e \in S$ and all $b_{0}, b_{1} \in T$ such that $\mu(e)=b_{0} \vee b_{1}$, there are elements $a_{0}$ and $a_{1}$ of $S$ such that $\mu\left(a_{0}\right) \leq b_{0}, \mu\left(a_{1}\right) \leq b_{1}$ and $e=a_{0} \vee a_{1}$.

For every non negative integer $n$, we will identify $n$ with the finite set (initial ordinal) $\{0,1, \ldots, n-1\}$.

\section{Congruence SPlitting LAttices; Uniform Refinement Properties}

We shall recall in this section some of the definitions and results of $[8,9]$ as well as a few new ones. Recall first [9, Section 3] that a lattice $L$ is congruence splitting when for all $a \leq b$ in $L$ and all congruences $\boldsymbol{a}_{0}$ and $\boldsymbol{a}_{1}$ in $L$, if $\Theta(a, b)=\boldsymbol{a}_{0} \vee \boldsymbol{a}_{1}$, then there exist elements $x_{0}$ and $x_{1}$ of $[a, b]$ such that $x_{0} \vee x_{1}=b$ and, for all $i<2$, $\Theta\left(a, x_{i}\right) \subseteq \boldsymbol{a}_{i}$. 
In [9, Proposition 3.2], we give a list of sufficient conditions for a lattice to be congruence splitting; this can be recorded here in the following fashion:

Proposition 1.1. The following holds:

(a) Every lattice that is either relatively complemented or sectionally complemented is congruence splitting.

(b) Every atomistic lattice is congruence splitting.

(c) The class of congruence splitting lattices is closed under direct limits.

There are easy examples of non congruence splitting lattices, as for example any chain with at least three elements. However, it is to be noted that two lattices may have isomorphic congruence lattices while one is congruence splitting and the other one is not. Our next definition is related to the effect of the congruence splitting property on the congruence lattice alone.

Definition 1.2. (see [9, Definition 2.1]) Let $S$ be a semilattice, let $e$ be an element of $S$. Say that the uniform refinement property (URP) holds at $e$ when for all families $\left(a_{i}\right)_{i \in I}$ and $\left(b_{i}\right)_{i \in I}$ of elements of $S$ such that $a_{i} \vee b_{i}=e($ all $i \in I$ ), there are families $\left(a_{i}^{*}\right)_{i \in I},\left(b_{i}^{*}\right)_{i \in I}$ and $\left(c_{i j}\right)_{(i, j) \in I \times I}$ of elements of $S$ such that for all $i, j, k \in I$, we have

(i) $a_{i}^{*} \leq a_{i}$ and $b_{i}^{*} \leq b_{i}$ and $a_{i}^{*} \vee b_{i}^{*}=e$.

(ii) $c_{i j} \leq a_{i}^{*}, b_{j}^{*}$ and $a_{i}^{*} \leq a_{j}^{*} \vee c_{i j}$.

(iii) $c_{i k} \leq c_{i j} \vee c_{j k}$.

Say that $S$ satisfies the URP when the URP holds at every element of $S$.

Then define similarly the weak uniform refinement property (WURP) at e when, under the same hypotheses on the $a_{i}, b_{i}(i \in I)$ and $e$, there are $c_{i j}$ such that for all $i, j, k \in I$, we have

(i') $c_{i j} \leq a_{i}, b_{j}$.

(ii') $c_{i j} \vee a_{j} \vee b_{i}=e$.

(iii') $c_{i k} \leq c_{i j} \vee c_{j k}$.

Say that $S$ satisfies the WURP when the WURP holds at every element of $S$.

Lemma 1.3. In the context above, the URP implies the WURP.

Proposition 1.4. Every distributive lattice satisfies the URP.

Proof. Let $\left(a_{i}\right)_{i \in I}$ and $\left(b_{i}\right)_{i \in I}$ two families of elements of a distributive lattice $D$ such that $a_{i}+b_{i}=$ constant. It is easy to verify that the elements $a_{i}^{*}=a_{i}, b_{i}^{*}=b_{i}$ and $c_{i j}=a_{i} \wedge b_{j}$ are as required.

We will need later the following straightforward lemma (see also [9, Proposition 2.3] for the URP):

Lemma 1.5. Let $\mu: S \rightarrow T$ be a weak-distributive homomorphism of semilattices and let $e$ be an element of $S$. If URP (resp. WURP) holds at e in $S$, then it also holds at $\mu(e)$ in $T$.

Corollary 1.6. Let $S$ be a distributive semilattice. If $S$ is the image of a distributive lattice by a weak-distributive homomorphism, then $S$ satisfies the URP (thus the WURP).

In particular, any distributive semilattice that is the image of a generalized Boolean algebra under a weak-distributive homomorphism (this is E. T. Schmidt's 
sufficient condition for being isomorphic to the congruence semilattice of a lattice, $[6,7])$ satisfies the WURP.

We end this section by recording one of the main results of [9]:

Theorem 1.7. [9, Theorem 3.3] Let $L$ be a congruence splitting lattice. Then $\mathbf{C}(L)$ satisfies the URP (thus the WURP).

Hence, by Lemma 1.3, if $L$ is a congruence splitting lattice, then $\mathbf{C}(L)$ also has the WURP. In particular, if we manage to find a lattice such that its congruence semilattice does not satisfy the WURP, then this lattice cannot be embedded congruence-preservingly into a congruence splitting lattice.

\section{The Decorations of $M_{3}$ AnD $N_{5}$}

From now on until Theorem 3.3, we shall fix a non distributive lattice variety $\mathcal{V}$. Let $\mathfrak{C}_{2}$ denote the two-element chain. For every set $X$, denote by $\mathbf{E}(X)$ the free product (=coproduct) of $X$ copies of $\mathfrak{C}_{2}$ in $\mathcal{V}$. Denote by $\mathbf{B}(X)$ the bounded lattice obtained from $\mathbf{E}(X)$ by adding a new largest element 1 and a new least element 0; write $\mathbf{E}_{\mathcal{V}}(X)$ (resp. $\mathbf{B}_{\mathcal{V}}(X)$ ) if $\mathcal{V}$ needs to be specified. Thus $\mathbf{B}(X)$ is generated as a bounded lattice by chains $s_{i}<t_{i}($ all $i \in X)$. Note that if $Y$ is a subset of $X$, then there is a canonical retraction from $\mathbf{B}(X)$ onto $\mathbf{B}(Y)$, sending each $s_{i}$ (resp. $t_{i}$ ) to 0 for every $i \in X \backslash Y$. Thus, we shall often identify $\mathbf{B}(Y)$ with the bounded sublattice of $\mathbf{B}(X)$ generated by all $s_{i}$ and $t_{i}(i \in Y)$. Moreover, the abovementioned retraction from $\mathbf{B}(X)$ onto $\mathbf{B}(Y)$ induces a retraction from $\mathbf{C}(\mathbf{B}(X))$ onto $\mathbf{C}(\mathbf{B}(Y))$. Hence, we shall also identify $\mathbf{C}(\mathbf{B}(Y))$ with the corresponding subsemilattice of $\mathbf{C}(\mathbf{B}(X))$.

From now on until Theorem 3.3, we shall fix a set $X$ such that $|X| \geq \aleph_{2}$. We denote, for all $i \in X$, by $\boldsymbol{a}_{i}$ and $\boldsymbol{b}_{i}$ the compact congruences of $\mathbf{B}(X)$ defined by

$$
\boldsymbol{a}_{i}=\Theta\left(0, s_{i}\right) \vee \Theta\left(t_{i}, 1\right) ; \quad \boldsymbol{b}_{i}=\Theta\left(s_{i}, t_{i}\right) .
$$

In particular, note that $\boldsymbol{a}_{i} \vee \boldsymbol{b}_{i}=\mathbf{1}$. Now, towards a contradiction, suppose that there are compact congruences $\boldsymbol{c}_{i j}(i, j \in X)$ such that for all $i, j, k \in X$, the following holds:

$$
\begin{gathered}
\boldsymbol{c}_{i j} \subseteq \boldsymbol{a}_{i}, \boldsymbol{b}_{j} \\
\boldsymbol{c}_{i j} \vee \boldsymbol{a}_{j} \vee \boldsymbol{b}_{i}=\mathbf{1} \\
\boldsymbol{c}_{i k} \subseteq \boldsymbol{c}_{i j} \vee \boldsymbol{c}_{j k} .
\end{gathered}
$$

Since the $\mathbf{C}$ functor preserves direct limits, there exists, for all $i, j \in X$, a finite subset $U=F(\{i, j\})$ of $X$ such that both $\boldsymbol{c}_{i j}$ and $\boldsymbol{c}_{j i}$ belong to $\mathbf{C}(\mathbf{B}(U))$. By Kuratowski's Theorem, there are mutually distinct elements, which we may denote by $0,1,2$ of $X$ such that $0 \notin F(\{1,2\}), 1 \notin F(\{0,2\})$, and $2 \notin F(\{0,1\})$. Let $\pi: \mathbf{B}(X) \rightarrow \mathbf{B}(3)$ be the canonical retraction. For every $i<3$, denote by $i^{\prime}$ and $i^{\prime \prime}$ the other two elements of 3 , arranged in such a way that $i^{\prime}<i^{\prime \prime}$. For all $i<3$, put $\boldsymbol{d}_{i}=\mathbf{C}(\pi)\left(\boldsymbol{c}_{i^{\prime} i^{\prime \prime}}\right)$.

Therefore, applying the semilattice homomorphism $\mathbf{C}(\pi)$ to the inequalities $(2.2$ -2.4 ) yields

$$
\begin{gathered}
\boldsymbol{d}_{0} \subseteq \boldsymbol{a}_{1}, \boldsymbol{b}_{2} ; \quad \boldsymbol{d}_{1} \subseteq \boldsymbol{a}_{0}, \boldsymbol{b}_{2} ; \quad \boldsymbol{d}_{2} \subseteq \boldsymbol{a}_{0}, \boldsymbol{b}_{1} ; \\
\boldsymbol{d}_{0} \vee \boldsymbol{a}_{2} \vee \boldsymbol{b}_{1}=\boldsymbol{d}_{1} \vee \boldsymbol{a}_{2} \vee \boldsymbol{b}_{0}=\boldsymbol{d}_{2} \vee \boldsymbol{a}_{1} \vee \boldsymbol{b}_{0}=\mathbf{1} ; \\
\boldsymbol{d}_{1} \subseteq \boldsymbol{d}_{0} \vee \boldsymbol{d}_{2} .
\end{gathered}
$$


Lemma 2.1. For all $i<3, \boldsymbol{d}_{i}$ belongs to $\mathbf{C}(\mathbf{B}(3 \backslash\{i\}))$.

Proof. For example for $i=0$. Since $0 \notin F(\{1,2\}), \boldsymbol{c}_{12}$ belongs to $\mathbf{B}(X \backslash\{0\})$, hence $\boldsymbol{d}_{0} \in \mathbf{B}(\{1,2\})$.

Now, since $\mathcal{V}$ is a non distributive variety of lattices, by a classical result of lattice theory, either the diamond $M_{3}$ or the pentagon $N_{5}$ belongs to the variety $\mathcal{V}$. Denote by $M$ one of these lattices that belongs to $\mathcal{V}$ and decorate it with three 2-element chains $x_{i}<y_{i}(i<3)$ in the following way:

Case 1. $M=M_{3}$. Let $p, q, r$ be the three atoms of $M_{3}$. Put

$$
\begin{array}{ll}
x_{0}=0 & y_{0}=p \\
x_{1}=q & y_{1}=1 \\
x_{2}=0 & y_{2}=r
\end{array}
$$

Case 2. $M=N_{5}$. Let $a>c$ and $b$ be the three join-irreducible elements of $N_{5}$. Put

$$
\begin{array}{ll}
x_{0}=0 & y_{0}=c \\
x_{1}=b & y_{1}=1 \\
x_{2}=0 & y_{2}=a
\end{array}
$$

Both cases can be described by the following picture:

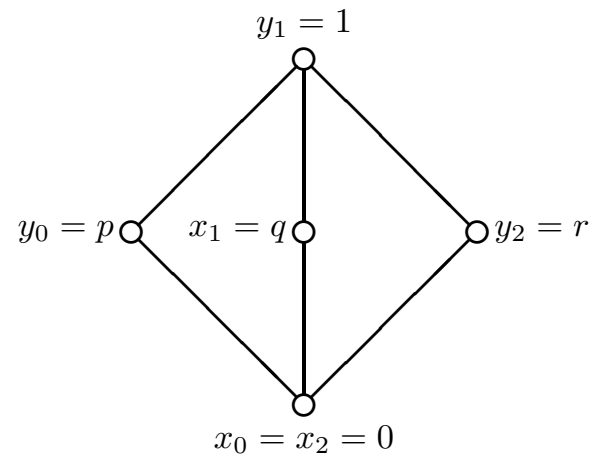

Case 1. $M=M_{3}$

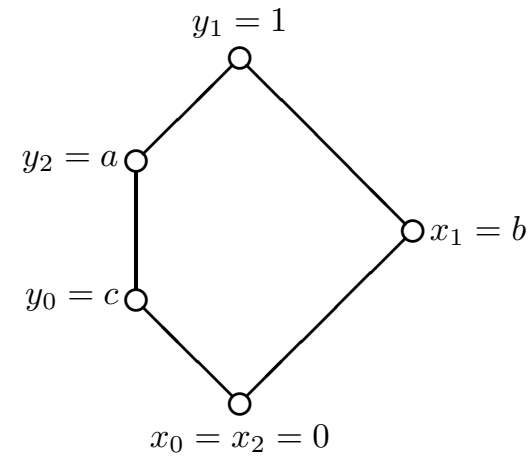

Case 2. $M=N_{5}$

The relevant properties of these decorations are summarized in both following straightforward lemmas:

Lemma 2.2. The decorations defined above satisfy the following inequalities

$$
x_{0} \wedge y_{1} \leq x_{1} ; \quad y_{1} \leq x_{1} \vee y_{0} ; \quad x_{1} \wedge y_{2} \leq x_{2} ; \quad y_{2} \leq x_{2} \vee y_{1},
$$

but $y_{2} \not \leq x_{2} \vee y_{0}$.

Lemma 2.3. For all $i<3$, the sublattice of $M$ generated by the elements $x_{j}$ and $y_{j}(j \neq i)$ is distributive.

This, along with $(2.5-2.7)$, will be sufficient to obtain a contradiction. Note already that since the free product of three 2-element chains in the variety generated by either $M_{3}$ or $N_{5}$ is finite, the problem is already reduced to a "computable" 
level. However, the size of the corresponding computations is such that it is useful to reduce (greatly) their complexity to mere computations in $M_{3}$ and $N_{5}$. This is what we shall do in Section 3.

\section{Reduction to THE Distributive WORLD}

From now on, we shall denote by $D$ be the free product of two 2-element chains in the variety of all distributive lattices. Thus, $D$ is generated by two chains $u_{0}<v_{0}$ and $u_{1}<v_{1}$.

Thus $D$ is a finite distributive lattice, that can be represented by the following diagram:

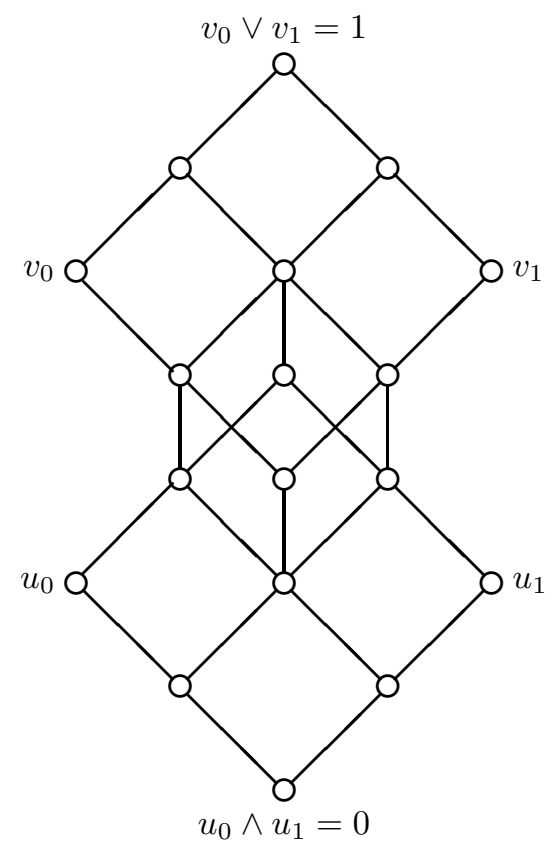

The lattice $D$

For all $i<3$, let $\pi_{i}: \mathbf{B}(3 \backslash\{i\}) \rightarrow D$ be the unique lattice homomorphism sending $s_{i^{\prime}}$ to $u_{0}, t_{i^{\prime}}$ to $v_{0}, s_{i^{\prime \prime}}$ to $u_{1}, t_{i^{\prime \prime}}$ to $v_{1}$. Furthermore, let $\rho: \mathbf{B}(3) \rightarrow M$ be the unique lattice homomorphism sending $s_{i}$ to $x_{i}$ and $t_{i}$ to $y_{i}($ all $i<3)$; let $\rho_{i}$ be the restriction of $\rho$ to $\mathbf{B}(3 \backslash\{i\})$.

Lemma 3.1. Let $L$ be any distributive lattice, let $a, b, a^{\prime}, b^{\prime}$ be elements of $L$. Then we have

$$
\Theta^{+}(a, b) \cap \Theta^{+}\left(a^{\prime}, b^{\prime}\right)=\Theta^{+}\left(a \wedge a^{\prime}, b \vee b^{\prime}\right) .
$$

Proof. Let $B$ be the generalized Boolean algebra R-generated by $L$ (in the sense of $[2$, Part II, Section 4]); identify every congruence $\theta$ on $L$ with the unique congruence on $B$ extending $\theta$. For all elements $x$ and $y$ of $B$, denote by $x \backslash y$ the unique relative complement of $x \wedge y$ in the interval $[0, x]$, and then put $x \triangle y=(x \backslash y) \vee(y \backslash x)$. Then a pair $(x, y)$ belongs to $\Theta^{+}(a, b)$ (resp. $\left.\Theta^{+}\left(a^{\prime}, b^{\prime}\right)\right)$ if and only if $x \Delta y \leq a \backslash b$ 
(resp. $\left.x \triangle y \leq a^{\prime} \backslash b^{\prime}\right)$. Therefore, $(x, y)$ belongs to $\Theta^{+}(a, b) \cap \Theta^{+}\left(a^{\prime}, b^{\prime}\right)$ if and only if $x \triangle y \leq(a \backslash b) \wedge\left(a^{\prime} \backslash b^{\prime}\right)=\left(a \wedge a^{\prime}\right) \backslash\left(b \vee b^{\prime}\right)$.

Remark. In particular, one recovers the classical result that if $a \leq b \leq c \leq d$ are elements of any distributive lattice, then $\Theta(a, b) \cap \Theta(c, d)=\mathbf{0}$.

Now, for all $i<3$, put $\boldsymbol{e}_{i}=\mathbf{C}\left(\pi_{i}\right)\left(\boldsymbol{d}_{i}\right)$.

Lemma 3.2. For all $i<3, \boldsymbol{e}_{i}=\Theta^{+}\left(u_{0} \wedge v_{1}, u_{1}\right) \vee \Theta^{+}\left(v_{1}, u_{1} \vee v_{0}\right)$.

Proof. Applying $\mathbf{C}\left(\pi_{i}\right)$ to the inequalities (2.5) and (2.6) yields both following inequalities:

$$
\begin{gathered}
\boldsymbol{e}_{i} \subseteq \Theta\left(0, u_{0}\right) \vee \Theta\left(v_{0}, 1\right) \text { and } \quad \boldsymbol{e}_{i} \subseteq \Theta\left(u_{1}, v_{1}\right) \\
\boldsymbol{e}_{i} \vee \Theta\left(0, u_{1}\right) \vee \Theta\left(v_{1}, 1\right) \vee \Theta\left(u_{0}, v_{0}\right)=\mathbf{1} .
\end{gathered}
$$

However, $D$ is a finite distributive lattice, thus $\mathbf{C}(D)$ is a finite Boolean algebra, and, by the Remark above, for all $j<2$, the elements $\Theta\left(0, u_{j}\right) \vee \Theta\left(v_{j}, 1\right)$ and $\Theta\left(u_{j}, v_{j}\right)$ are complemented elements of $\mathbf{C}(D)$; in fact, $\mathbf{1}$ is the disjoint union of $\Theta\left(0, u_{j}\right), \Theta\left(u_{j}, v_{j}\right), \Theta\left(v_{j}, 1\right)$. Then, from both inequalities (3.1) and (3.2) and a new application of Lemma 3.1, one deduces easily that

$$
\begin{aligned}
\boldsymbol{e}_{i} & =\left(\Theta\left(0, u_{0}\right) \vee \Theta\left(v_{0}, 1\right)\right) \cap \Theta\left(u_{1}, v_{1}\right) \\
& =\left(\Theta\left(0, u_{0}\right) \cap \Theta\left(u_{1}, v_{1}\right)\right) \vee\left(\Theta\left(v_{0}, 1\right) \cap \Theta\left(u_{1}, v_{1}\right)\right) \\
& =\Theta^{+}\left(u_{0} \wedge v_{1}, u_{1}\right) \vee \Theta^{+}\left(v_{1}, u_{1} \vee v_{0}\right) .
\end{aligned}
$$

Now, for all $i<3$, it results from Lemma 2.3 that there exists a unique lattice homomorphism $\varphi_{i}: D \rightarrow M$ such that $\varphi_{i} \circ \pi_{i}=\rho_{i}$. The corresponding commutative diagram is the following:

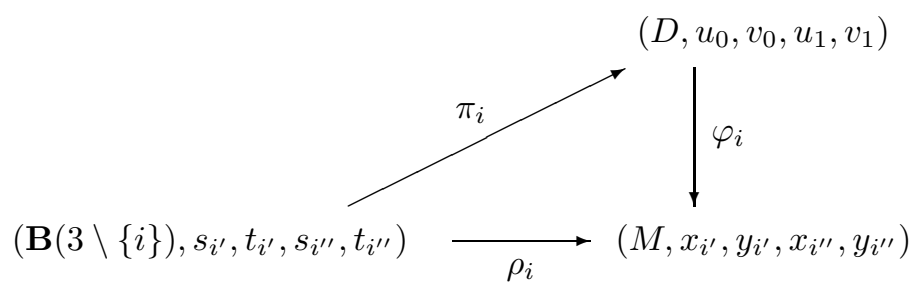

Since $\mathbf{C}$ is a functor, we get from this and from Lemma 3.2 that for all $i<3$, we have

$$
\begin{aligned}
\mathbf{C}(\rho)\left(\boldsymbol{d}_{i}\right)=\mathbf{C}\left(\varphi_{i}\right)\left(\boldsymbol{e}_{i}\right)=\mathbf{C}\left(\varphi_{i}\right) & \left(\Theta^{+}\left(u_{0} \wedge v_{1}, u_{1}\right) \vee \Theta^{+}\left(v_{1}, u_{1} \vee v_{0}\right)\right) \\
& =\Theta^{+}\left(x_{i^{\prime}} \wedge y_{i^{\prime \prime}}, x_{i^{\prime \prime}}\right) \vee \Theta^{+}\left(y_{i^{\prime \prime}}, x_{i^{\prime \prime}} \vee y_{i^{\prime}}\right) .
\end{aligned}
$$

In particular, we have, using Lemma 2.2,

$$
\begin{aligned}
\mathbf{C}(\rho)\left(\boldsymbol{d}_{0}\right) & =\Theta^{+}\left(x_{1} \wedge y_{2}, x_{2}\right) \vee \Theta^{+}\left(y_{2}, x_{2} \vee y_{1}\right)=\mathbf{0}, \\
\mathbf{C}(\rho)\left(\boldsymbol{d}_{2}\right) & =\Theta^{+}\left(x_{0} \wedge y_{1}, x_{1}\right) \vee \Theta^{+}\left(y_{1}, x_{1} \vee y_{0}\right)=\mathbf{0}, \\
\text { but } \quad & \mathbf{C}(\rho)\left(\boldsymbol{d}_{1}\right)=\Theta^{+}\left(x_{0} \wedge y_{2}, x_{2}\right) \vee \Theta^{+}\left(y_{2}, x_{2} \vee y_{0}\right) \neq \mathbf{0} .
\end{aligned}
$$

On the other hand, by applying $\mathbf{C}(\rho)$ to (2.7), we obtain that

$$
\mathbf{C}(\rho)\left(\boldsymbol{d}_{1}\right) \leq \mathbf{C}(\rho)\left(\boldsymbol{d}_{0}\right) \vee \mathbf{C}(\rho)\left(\boldsymbol{d}_{2}\right),
$$


a contradiction. Therefore, we have proved the following theorem:

Theorem 3.3. Let $\mathcal{V}$ be any non distributive variety of lattices, let $X$ be any set such that $|X| \geq \aleph_{2}$. Let $\mathbf{B}_{\mathcal{V}}(X)$ be the free product in $\mathcal{V}$ of $X$ copies of a 2-element chain with a least and a largest element added. Then $\mathbf{C}\left(\mathbf{B}_{\mathcal{V}}(X)\right)$ does not satisfy WURP at $\mathbf{1}$.

\section{EXTENSIONS TO FURTHER LATTICES AND TO REGULAR RINGS}

This section will be devoted to harvest consequences of Theorem 3.3.

Corollary 4.1. Let $L$ be any lattice that admits a lattice homomorphism onto a free bounded lattice in the variety generated by either $M_{3}$ or $N_{5}$ with $\aleph_{2}$ generators. Then $\mathbf{C}(L)$ does not satisfy WURP at $\mathbf{1}$. In particular, there exists no congruence splitting lattice $K$ such that $\mathbf{C}(K) \cong \mathbf{C}(L)$; furthermore, $\mathbf{C}(L)$ does not satisfy Schmidt's condition.

Proof. Let $\mathcal{V}$ be the lattice variety generated by either $M_{3}$ or $N_{5}$ and, for any set $X$, let $\mathbf{F}_{\mathcal{V}}(X)$ be the free bounded lattice on $X$ in $\mathcal{V}$. First, if the cardinality of $X$ is infinite, then there exists a surjective lattice homomorphism from $\mathbf{F}_{\mathcal{V}}(X)$ onto $\mathbf{B}_{\mathcal{V}}(X)$ (split $X$ into two disjoint sets $X_{0}$ and $X_{1}$ such that $\left|X_{0}\right|=\left|X_{1}\right|=|X|$; send the elements of $X_{1}\left(\operatorname{resp} . X_{2}\right)$ onto all $s_{i}\left(\right.$ resp. $\left.\left.t_{i}\right)\right)$. Therefore, if $|X|=\aleph_{2}$, then there exists by assumption a surjective lattice homomorphism $f: L \rightarrow \mathbf{B}_{\mathcal{V}}(X)$. By [9, Proposition 1.2], the corresponding congruence mapping $\mathbf{C}(f): \mathbf{C}(L) \rightarrow$ $\mathbf{C}\left(\mathbf{B}_{\mathcal{V}}(X)\right)$ is weak-distributive. Thus, if $\mathbf{C}(L)$ would satisfy the WURP at $\mathbf{1}$, then, by Lemma 1.5, $\mathbf{C}\left(\mathbf{B}_{\mathcal{V}}(X)\right)$ would also satisfy the same refinement property, therefore contradicting Theorem 3.3. The last two statements result from Theorem 1.7 and Corollary 1.6.

This shows in particular that there are distributive semilattices that are representable as congruence semilattices of lattices (the $\mathbf{C}(L)$ 's, with $L$ free lattice on at least $\aleph_{2}$ generators in any non-distributive variety) that, nevertheless, do not satisfy any of the known sufficient conditions implying representability (as Schmidt's condition).

Corollary 4.2. Let $\mathcal{V}$ be any non distributive variety of lattices and let $F$ be any free (resp. free bounded) lattice with at least $\aleph_{2}$ generators in $\mathcal{V}$. Then there exists no congruence splitting lattice $K$ such that $\mathbf{C}(K) \cong \mathbf{C}(F)$.

Corollary 4.3. Let $\mathcal{V}$ and $F$ as above. Then there exists no von Neumann regular ring $R$ whose semilattice of finitely generated two-sided ideals is isomorphic to $\mathbf{C}(F)$.

Proof. Proposition 1.1 (and the fact that the lattice of principal right ideals of $R$ is sectionally complemented), Corollary 4.2 and [9, Corollary 4.4].

We do not know whether every lattice of cardinality at most $\aleph_{1}$ admits a congruence-preserving extension into a sectionally complemented lattice. On the other hand, G. Grätzer and E. T. Schmidt prove in [4] that every finite lattice has a congruence-preserving extension into a sectionally complemented finite lattice. Note that if $\mathcal{V}$ is a non distributive lattice variety generated by a single finite lattice, then $\mathbf{F}_{\mathcal{V}}(X)$ is a direct limit of a limit system of finite lattices with embeddings having the congruence extension property; nevertheless, its congruence semilattice is complicated in the sense that it does not satisfy WURP. 


\section{ACKNOWLEDGMENTS}

The authors wish to thank warmly Ralph Freese who, when questioned about it, returned to them very quickly the construction of the lattice freely generated by two 2-element chains in the pentagon variety. Although its use has eventually been eliminated in the final version of the proof, it greatly helped in its elaboration. The paper was completed while the second author was visiting the University of Caen. The hospitality and excellent conditions provided by the mathematics department are greatly appreciated.

\section{REFERENCES}

[1] G. M. Bergman, "Von Neumann regular rings with tailor-made ideal lattices", unpublished notes, October 1986.

[2] G. Grätzer, General Lattice Theory, Pure and Applied Mathematics 75, Academic Press, Inc. (Harcourt Brace Jovanovich, Publishers), New York-London; Lehrbücher und Monographien aus dem Gebiete der Exakten Wissenschaften, Mathematische Reihe, Band 52. Birkhäuser Verlag, Basel-Stuttgart; Akademie Verlag, Berlin, 1978. xiii+381 pp.

[3] G. Grätzer and E. T. Schmidt, "On congruence lattices of lattices", Acta Math. Sci. Hungar. 13 (1962), pp. 179-185.

[4] G. Grätzer and E. T. Schmidt, "Congruence-preserving extensions of finite lattices to sectionally complemented lattices", to appear in Proc. Amer. Math. Soc.

[5] C. Kuratowski, "Sur une caractérisation des alephs", Fund. Math. 38 (1951), pp. 14-17.

[6] E. T. Schmidt, "Zur Charakterisierung der Kongruenzverbände der Verbände", Mat. Časopis Sloven. Akad. Vied 18 (1968), pp. 3-20.

[7] M. Tischendorf, "On the representation of distributive semilattices", Algebra Universalis 31 (1994), pp. 446-455.

[8] F. Wehrung, "Non-measurability properties of interpolation vector spaces", Israel Journal of Mathematics 103 (1998), pp. 177-206.

[9] F. Wehrung, "A uniform refinement property of certain congruence lattices", Proc. Amer. Math. Soc. 127, no. 2 (1999), pp. 363-370.

Mathematical Institute, Slovak Academy of Sciences, Grešákova 6, 04001 Košice, SLOVAKIA

E-mail address: ploscica@linux1.saske.sk

Department of Algebra, Faculty of Mathematics and Physics, Sokolovská 83, Charles University, 18600 Praha 8, Czech Republic

E-mail address: tuma@karlin.mff.cuni.cz

C.N.R.S., Département de Mathématiques, Université de Caen, 14032 Caen Cedex, FranCE

E-mail address: gremlin@math.unicaen.fr 